\title{
Paisaje cultural cafetero del Tolima: una construcción social del patrimonio
}

\section{Coffee region"s culltural landscape of Tolima, Colombia: a social construction of heritage}

\author{
César Augusto Velandia Silva ${ }^{1}$ \\ Sergio Andrés Forero Machado?
}

(.)

Recepción: 18/08/2020
(2)

Aprobación: 02/09/2020
(-)

Publicación: 18/12/2020

\section{Para citar este artículo:}

Velandia Silva, C.A, \& Forero Machado, S. A. Paisaje cultural cafetero del

Tolima: una construcción social del patrimonio. Indagare, (8), 78-99.

https://doi.org/10.35707/indagare/808

\section{(c) $(9)$}

\footnotetext{
${ }^{1}$ Grupo de investigación Rastro Urbano. Universidad de Ibagué, Colombia. ORCID: 0000-0003-0187-6488. Correo electrónico: cesar.velandia@unibague.edu.co

${ }^{2}$ Grupo de investigación Rastro Urbano. Universidad de Ibagué, Colombia. ORCID: 0000-0002-6362-4078. Correo electrónico: sergio.forero@unibague.edu.co
} 


\section{Resumen}

El artículo elabora la puesta en valor del paisaje cultural cafetero del Tolima mediante la definición de sus valores, atributos y su delimitación. A partir de esto, propone una agenda social para abarcar, validar y mejorar los componentes con las comunidades $y$, finalmente, identifica temas claves específicos para la gestión, con base en estrategias y herramientas de participación con las comunidades, de manera que los grupos sociales asuman su responsabilidad en la valoración del paisaje. Este trabajo representa el primer enfoque desarrollado acerca de la valorización del paisaje cultural cafetero del Tolima y ofrece las claves para el manejo de aspectos clave para la conservación del sitio.

\section{Abstract}

This article aims the enhancement of the coffee region's cultural landscape of Tolima by defining its values, attributes and its delimitation. From that, it proposes a social agenda to encompass, validate and improve the components with communities and, finally, identifies specific key issues for management, based on strategies and tools for participation with communities, so that social groups assume their responsibility in the valuation of the landscape. This work represents the first approach developed about the appreciation of the coffee region's cultural landscape of Tolima and provides the clues for the management of key aspects for the conservation of the site.

\footnotetext{
Palabras claves: Paisaje cultural, café del Tolima, patrimonio cultural.
Key words: Cultural landscape, coffee from Tolima, cultural heritage.
}

\section{Introducción}

Desde la inclusión de los paisajes culturales en 1992 en la Convención del Patrimonio Mundial (Unesco, 2017) y la inscripción de sitios en la Lista del Patrimonio Mundial, han surgido varios temas de debate respecto a cómo se gestionan los paisajes, así como los retos a los que se enfrentan estos territorios distinguidos por su valor universal excepcional. Los organismos asesores de la Unesco (ICCROM, ICOMOS, UICN), han profundizado sobre las relaciones entre la naturaleza y la cultura, el manejo y gestión de sitios (Larsen y Wijesuriya, 2015; Markham, 2018; Plachter y Rössler, 1995; Rössler, 1993, 2003, Rössler y Tricaud, 2009; Von Droste et al., 1995; Wagstaff, 1987). 
A pesar de lo anterior, en Latinoamérica, la metodología de valoración, significación y manejo del valor universal excepcional, en los paisajes culturales no se adapta a la alta diversidad, ni logra enfatizar las profundas relaciones que persisten en el paisaje simbólico, que requieren métodos más precisos para su delimitación, construcción social y manejo. Adicionalmente, existen grandes dificultades en la vinculación de la legislación internacional al compromiso obligatorio para el marco jurídico nacional o regional de cada país (Instituto Nacional de Patrimonio Cultural-INPC, 2014, 2015; Ministerio de Cultura del Perú, 2017; Mujica y Holle, 2002; Rincón, 2016; Uribe y Velandia, 2013).

Esta investigación presenta la primera propuesta de delimitación del paisaje cultural cafetero del Tolima, y pretende encontrar, además, cómo su metodología debe convertirse en una gestión con la comunidad y vincularse a un plan de manejo, que constituirá un insumo fundamental para la adopción del posterior programa de gestión y valoración con mayor profundidad. Si bien existe una política nacional de paisaje (Departamento Nacional de Planeación-DNP, 2014) aplicada solo al territorio inscrito en la Lista de Patrimonio Mundial de Unesco en 2011 (áreas de municipios de Caldas, Quindío, Risaralda y Valle del Cauca), debe aclararse que no existen actualmente iniciativas (públicas o privadas) dirigidas a una valoración y conservación del paisaje cultural cafetero del Tolima, y lo que es más complejo: no existe un conocimiento construido con las comunidades cafeteras para este fin.

La iniciativa del paisaje cultural cafetero del Tolima fue considerada durante el proceso de formulación del expediente de candidatura del Paisaje Cultural Cafetero de Colombia para la Unesco (s.f.). Sin embargo, los representantes del Tolima desistieron sobre su participación y posterior inclusión en la delimitación del bien.

Por lo tanto, nuestra región perdió la oportunidad de contar con una agenda de conservación del patrimonio en su territorio, que le permitiera aprovechar ventajas como la experiencia del Paisaje Cultural Cafetero de Colombia, la cual, sin precedentes en Colombia, estableció nuevos referentes sobre la valoración del patrimonio. Los retos de difusión, coordinación y gestión con los organismos y comunidades de la región de Caldas, Quindío, Risaralda y Valle del Cauca, fueron abordados por el Gobierno colombiano, que emprendió acciones específicas con la Federación Nacional de Cafeteros de Colombia (FNCC) y los gobiernos regionales para promover acciones de conservación (Ver Figura 1). 
INDAGARE e-ISSN: 2357-5042 • Número 8 (2020) • Universidad de Ibagué • doi: https://doi.org/10.35707/indagare/808

Figura 1. Paisaje cultural cafetero del Tolima

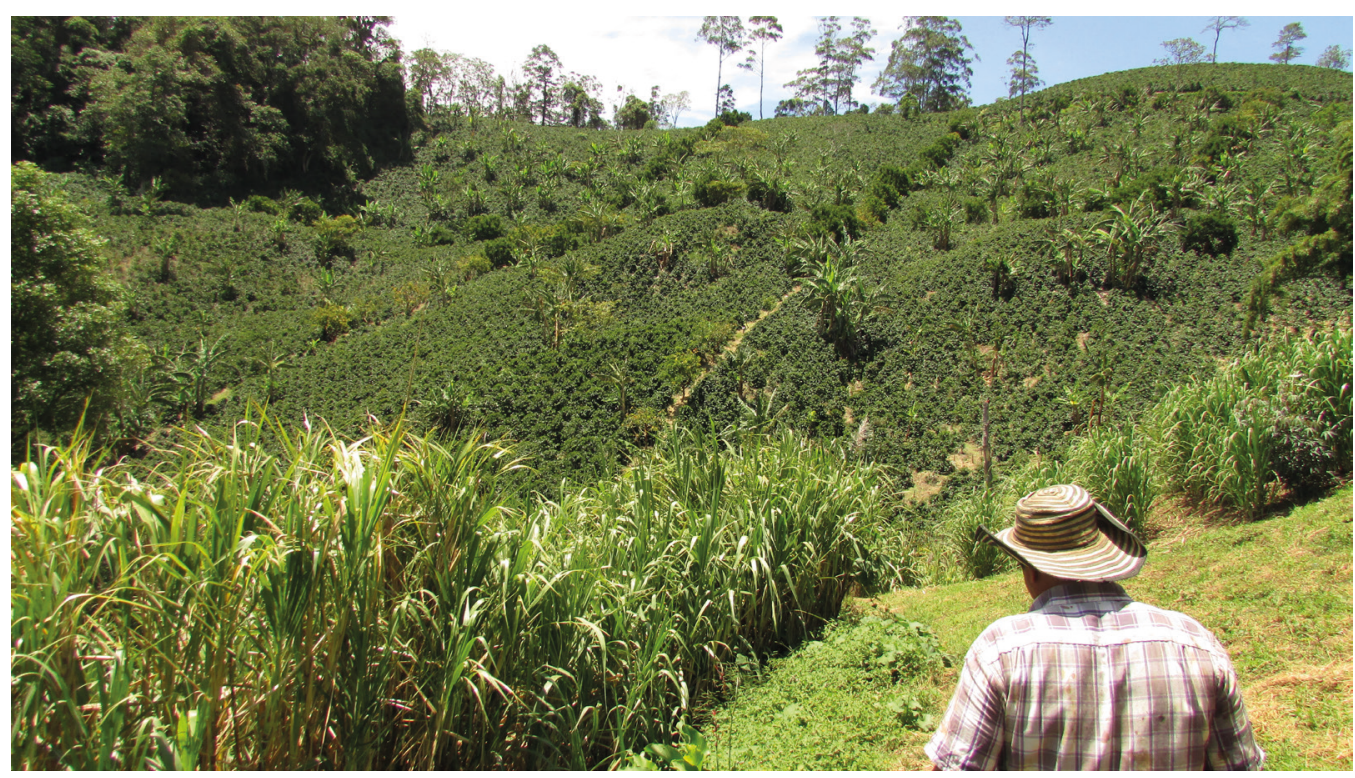

Fuente: Finca Las Américas. Municipio de San Juan de la China. Fotografía de Juan Sebastián García Zamora. Integrante del semillero de investigación Paisajes Culturales del Tolima (2020)

Considerando los actuales procesos de formulación de paisajes culturales (INPC, 2015; Ministerio de Cultura del Perú, 2017; Unesco, 2017), es posible desarrollar lineamientos propios para la construcción de un conocimiento sobre la "valoración del patrimonio cafetero del Tolima" (Velandia, 2018, p. 48).

A pesar de que Colombia tiene una definición inclusiva de patrimonio por ley, según Isaza (2014) el patrimonio encarna "los sitios naturales, los paisajes culturales e incluso las innumerables manifestaciones intangibles de la cultura actual de cualquier pueblo del mundo, independientemente de su grado de desarrollo cultural, económico, político o tecnológico" (p. 2). Esta visión inclusiva, diversa y participativa del patrimonio corresponde a la noción de patrimonio colombiano, tal como se define en la Ley de Cultura. De acuerdo con el artículo 4 de la Ley 1185 (República de Colombia, 2008) se constituye: 
[...] por todos los bienes materiales, manifestaciones inmateriales, productos y representaciones de la cultura que son expresión de la nacionalidad colombiana, tales como la lengua castellana, las lenguas y dialectos de las comunidades indígenas, negras y criollas, la tradición, los conocimientos ancestrales, el paisaje cultural, los usos y costumbres, así como los bienes materiales de naturaleza mueble o inmueble a los que se atribuye un especial interés histórico, artístico, científico, estético o simbólico, entre otros, en campos como el plástico, arquitectónico, urbano, arqueológico, lingüístico, sonoro, musical, audiovisual, fílmico, testimonial, documental, literario, bibliográfico, museológico o antropológico. (Ley 1185 de 2008, art. 1).

Según Unesco (2008), para definir el término "paisaje cultural”, se discutió el equilibrio entre el patrimonio cultural y el natural, como: "Las obras conjuntas del hombre y la naturaleza” (p.15), y también se lo denominó patrimonio cultural. Los paisajes culturales "ilustran la evolución de la sociedad humana y sus asentamientos a lo largo del tiempo, condicionados por las limitaciones y/o oportunidades físicas de su entorno natural y las sucesivas fuerzas sociales, económicas y culturales, tanto externas como internas" (p. 16). El término paisaje cultural "abarca una diversidad de manifestaciones de la interacción entre la humanidad y su entorno natural” (p. 96). Según el Comité de Patrimonio Mundial (Unesco, 2008), "debería seleccionarse sobre la base de su valor universal excepcional y su representatividad en términos de una región geocultural claramente definida y, por consiguiente, de su capacidad para ilustrar los elementos culturales esenciales y distintivos de esas regiones" (p. 96).

Recientemente, el Decreto 2358 de 2019 (Mincultura, 2019) redefinió el término "paisaje cultural" como:

Los territorios resultantes de la interrelación de grupos sociales, comunidades o colectivos con su territorio o naturaleza, refiriéndose a los procesos históricos, económicos, sociales, políticos, culturales o simbólicos, que ilustran las formas de ocupación y gestión del territorio. Por lo tanto, son factores de identidad, pertenencia o ciudadanía, y contienen bienes, manifestaciones, productos. Son expresiones de identidad cultural y son representativas de una región claramente definida, e ilustran los elementos culturales esenciales y distintivos; mediante la apreciación y la gestión sostenible de esos lugares, se hace efectivamente posible el disfrute de los derechos culturales. (Ministerio de Cultura, 2019, p. 48).

Existen siete categorías: 1) Claramente definido, concebido y creado intencionalmente por el hombre; 2) Evolucionado orgánicamente; 3) Relicto o fósil; 4) Viviente; 5) Asociativo; 6) Lugares de memoria; y 7) Complejos socioculturales. 
Pero en el contexto de los países de América Latina, este sistema no siempre permite una interpretación científica completa. La pregunta es: ¿Cuál es la parte cultural del paisaje? La relación hombre-naturaleza, las interrelaciones con la naturaleza y la cultura se expresan en un espacio simbólico-antropológico (Cleere, 1995; Cosgrove, 1984, 1985, 1994; Cosgrove y Daniels, 1988; Wagstaff, 1987). Este se refiere en un marco desde que los pueblos indígenas desarrollaron una comprensión del territorio al establecer espacios vitales, funerarios, agrícolas a través de pisos térmicos (debido a la zona ecuatorial) y amplios sistemas de comunicación de caminos a través de las cordilleras andinas.

Por lo tanto, los sistemas culturales son interacciones estructuradas y organizadas entre las personas y su entorno (Anschuetz et al., 2001; Deetz, 1990; Hirsch y O'Hanlon, 1995; Ingold, 1993). Como señala Cosgrove (1985), el paisaje significa un mundo exterior mediado por la experiencia subjetiva del hombre. Es el mediador entre la naturaleza y la cultura. Es una construcción, una composición de su mundo (Norton, 1989).

El estudio de las interrelaciones mencionadas se refiere a los aspectos "vivos" vinculantes entre sus dimensiones; concentrados y entrelazados en los valores simbólicos de las tradiciones y su diversidad biocultural (Larsen y Wijesuriya, 2015). Así, lo cultural es el rasgo "vivo" del paisaje. Un modelo para su comprensión "como expresión viva debe permanecer expuesto para la aprehensión social y para su preservación a través del tiempo" (Velandia, 2017, p. 49). Esto es muy significativo en el paisaje agrícola. De esta manera, el paisaje cultural es una construcción social de un territorio, basada en la transformación histórica de la naturaleza a través del trabajo colectivo y de modificaciones enriquecedoras como las prácticas de adaptación de diferentes cultivos como el maíz, la papa o el café. Las prácticas agrícolas en el territorio, están representadas por el trabajo étnico a lo largo del tiempo y localizadas en un espacio determinado, donde se manifiesta una visión dinámica del cambio cultural. De acuerdo con el Instituto Nacional de Patrimonio Cultural (INPC) (2015) el paisaje cultural es:

Una parte del territorio que comprende un sistema articulado de interacciones naturales y humanas integrado por su geografía y procesos históricos desarrollados produciendo espacios de características singulares con valores históricos, socioculturales, ecológicos, estéticos, visuales, productivos, económicos, religiosos y simbólicos de reconocimiento local, municipal, regional, nacional e internacional. (Instituto Nacional de Patrimonio Cultural, 2015, p. 29).

La comprensión de los orígenes del paisaje, sus patrones y los asentamientos de las fincas cafeteras dispersas en el territorio, presupone una explicación a partir de la teoría de la microverticalidad de Murra (1975). Los estudios sobre la ocupación del 
suelo en los Andes (Argüello, 2016; Cuéllar, 2009, 2011; Mujica y Holle, 2002; INPC, 2014; Quattrin, 2001) se basan en las "economías verticales" o el "archipiélago vertical" (Murra, 1974; 1975); a partir de las cuales se articulan y se asientan los grupos indígenas en las cordilleras andinas colombianas. La ocupación de la cordillera Central y Oriental está relacionada con un proceso de amplio conocimiento de los pisos térmicos y el uso de las zonas templadas para vivir y producir cultivos tradicionales de mayor o menor calidad, según un conocimiento y experiencia en el manejo del suelo y el agua.

Según Cuéllar (2009), la verticalidad serrana (Murra, 1974), se trata de un "modelo de complementariedad ecológica" (p. 8) desarrollado a partir de una estrategia de dominación de los cacicazgos (entendidos como unidades políticas) para el aprovechamiento de los ambientes altitudinales de la cordillera; con la finalidad de generar "una invariable autonomía microeconómica y social por parte de una sola etnia” (Cuéllar, 2011, p. 43), claramente presentada en los Andes del norte (páramo de Colombia), en los que los corredores interandinos son muy estrechos y en los que existe una mayor variabilidad ecológica expresada en parches contiguos de gran diversidad, lo cual permite a las familias acceder a diferentes zonas ecológicas y a una variada producción agrícola.

En las tierras bajas del valle del río Magdalena, los indígenas contaban con pesca y cultivos como la caña de azúcar y el maíz (Zea mays); en las zonas medias de clima templado tenían viviendas y cultivos de cítricos, plátanos, papas y frijoles en las tierras altas. Las pruebas arqueológicas indican que los viajes transversales a las cordilleras se hacían por antiguos caminos que originalmente recorrían por las orillas de las crestas en dirección contraria a los valles de los arroyos.

El paisaje cultural cafetero del Tolima es el resultado de 12000 años de actividad humana (Cano y López, 2017; Dickau et al., 2015; Salgado y Varón, 2019). Las evidencias arqueológicas de las prácticas agrícolas se localizan especialmente entre los 900 y 1800 metros de altitud que coincide con el espacio cafetero desarrollado posteriormente a finales del siglo XIX. Las fincas cafeteras fueron construidas sobre los espacios de vida, cultivo y funerarios desarrollados desde tiempos precolombinos (Ver Figura 2). 
Figura 2. Ubicación del paisaje cultural cafetero (inscrito en la Lista del Patrimonio Mundial) y del paisaje cultural cafetero del Tolima

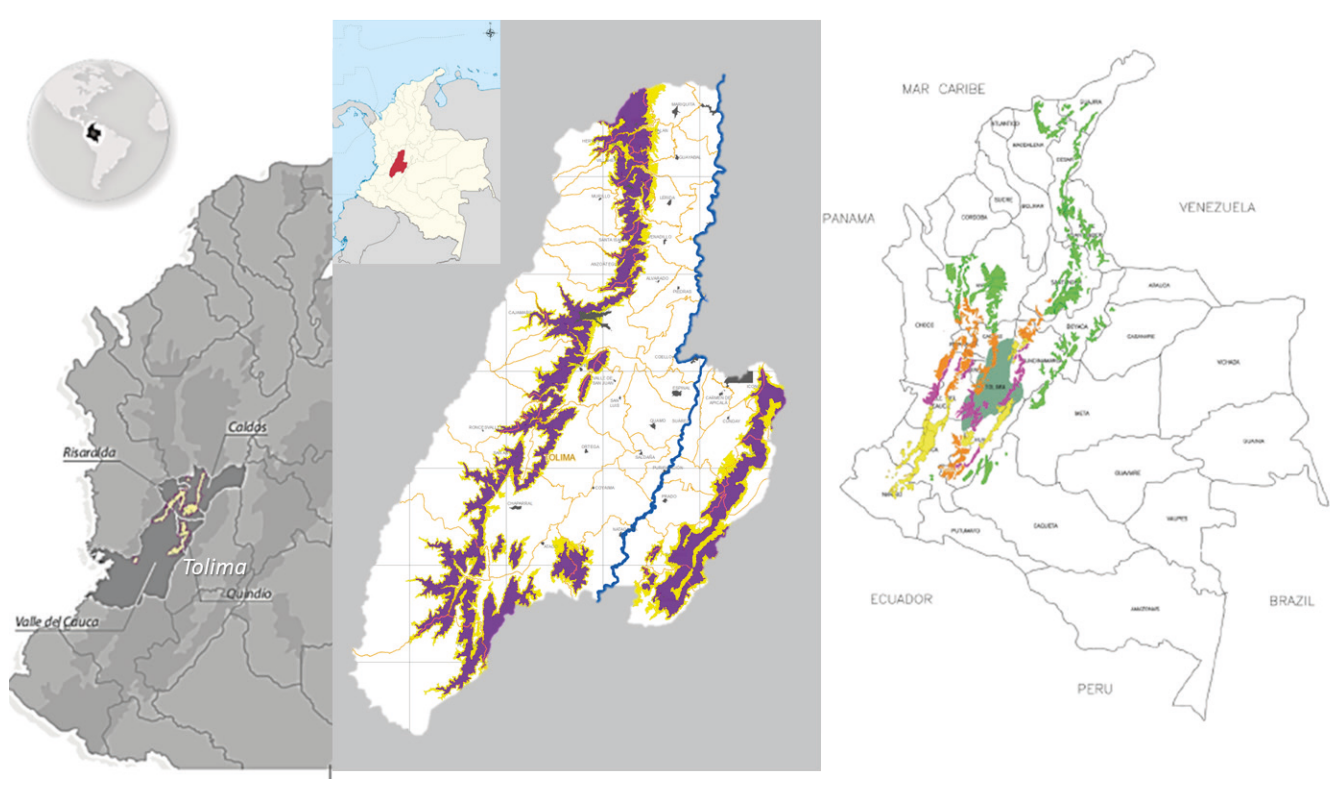

Fuente: Velandia (2018), Federación Nacional de Cafeteros de Colombia. FNCC (2015) y

Creative Commons Attribution-Share Alike 3.0

\section{Materiales y métodos}

El contexto geográfico del paisaje cultural cafetero del Tolima es muy similar al del caso del Paisaje Cultural Cafetero de Colombia de la Unesco: una muestra de un paisaje único, productivo y sostenible "que representa la tradición cafetera colombiana" (Velandia, 2017, p. 44). El paisaje cafetero, en sí mismo, es la manifestación de los vínculos entre la naturaleza y la cultura representados en una tradición centenaria, ilustrada "por la adaptación de los minifundios, el uso inteligente de las fuentes de agua, el desarrollo de materiales de construcción tradicionales, la comprensión simbólica del paisaje y la coexistencia con el riesgo de desastres" (Velandia, 2017, p. 44). Fue valorada por la Unesco debido al uso continuo de la tierra en el que los agricultores aplicaron prácticas innovadoras de gestión de los recursos naturales y produjeron las manifestaciones culturales tangibles e intangibles que caracterizan a la región.

A partir del procesamiento de una geodatabase del Comité de Cafeteros del Tolima (FNCC, 2019), de la caracterización de la denominación de origen (FNCC, 2015) y de la experiencia de gestión del plan de manejo del Paisaje Cultural Cafetero de Colombia, 
con el cual el autor tuvo la experiencia de trabajar en varios talleres con la comunidad (Mincultura, 2009, 2016). El enfoque de la Paisaje Cultural Cafetero de Colombia se definió a partir de la siguiente metodología:

- Identificación del contexto histórico y geográfico.

- Comprensión de los sistemas culturales para la transmisión de los conocimientos tradicionales por medio de la identificación de los atributos del paisaje (cualitativos y cuantitativos).

- Construcción de un modelo de delimitación a partir de atributos cuantitativos.

- Organizar la agenda social básica.

- Definir un programa de gestión de acuerdo con los aspectos clave para la gestión. La promoción, de una agenda posterior, puede basarse en los antecedentes de los procesos 2011-2019 del plan de gestión del Paisaje Cultural Cafetero de Colombia y la reflexión de sus experiencias (Corporación Autónoma Regional de Risaralda-Carder, 2002; Isaza y Velandia, 2018; Mincultura-FNCC, 2009, 2016; Rincón, 2016; Uribe y Velandia, 2013).

\subsection{Contexto histórico y geográfico del café del Tolima}

El café del Tolima es estratégico para la producción cafetera de Colombia. El Tolima es el tercer productor de Colombia y representa una de las seis denominaciones de origen de la FNCC (2019). Los departamentos del Paisaje Cultural Cafetero de Colombia (Eje Cafetero) tales como: Caldas, tiene el quinto lugar; Valle del Cauca, el sexto; Risaralda, el séptimo; y Quindío, el cual ni siquiera está entre los 10 primeros productores y carecen de denominación de origen (FNCC-Huila, s.f.).

De acuerdo con Palacios (1980), los holandeses introdujeron el arbusto del café en América del Sur con fines comerciales. En 1714 fue plantado en Surinam y desde allí se trasladó a Venezuela y Brasil. Guhl (2008) explica cómo el café se expandió a través de los departamentos de Santander y la región de Norte de Santander en Colombia, en las primeras décadas del siglo XIX (Velandia, 2018). Desde allí se extendió lentamente hacia el sur a lo largo de la cordillera Oriental.

En el período entre 1840 y 1860 llegó a Cundinamarca, Tolima, algunas partes del Huila y el Cauca, y su producción estuvo en manos de grandes terratenientes. En 1870 ya había llegado al departamento de Antioquia y entre 1880 y 1910; desde allí se extendió hacia el sur a los departamentos de Caldas, Risaralda, Quindío y al norte del Valle del Cauca. (Velandia, 2018, p. 49). 
La Federación Nacional de Cafeteros de Colombia (FNCC,2015), confirma que el café apareció en 1870 en los municipios de Venadillo, Santa Isabel y Rovira en Tolima, y se convirtió en dominante en la zona del Chaparral en el sur de Tolima (Palacios, 1980).

La rápida expansión territorial y la mayor importancia económico-social del café fueron notables, pues entre 1870 y 1910 se consolidó la economía cafetera. Esto implicó no solo un desplazamiento de las zonas de producción de café, sino que la presencia de estas nuevas formas de organización social y productiva se asoció a un crecimiento exponencial después de la guerra civil colombiana. En ese momento se tuvieron las tareas más arduas del asentamiento del cultivo en la cordillera Central. La región estaba contenida entre los suelos más fértiles y las condiciones más aptas del ambiente, que finalmente, se articula con una red de empresas comerciales y financieras sobre la base de la alta burguesía empresarial.

[Los procesos de] distribución de los terrenos baldíos, la migración interna y la inversión de capital urbano en las zonas de colonización, fueron procesos decisivos en la consolidación de la caficultura colombiana. La expansión de la industria cafetera fue el resultado de una especialización productiva en las estructuras agrarias. (Ramírez, 2008, p. 39).

Al mismo tiempo, entró en servicio la línea de transporte del Ferrocarril entre el puerto de Girardot y el puente sobre el Magdalena, que consolidó el papel como centro de distribución de una vasta región del centro de Tolima y Huila. Se establecieron casas comerciales, trilladoras y exportadores de café. El Tolima era en ese momento una de las zonas de producción más características de la industria cafetera (Hall et al., 1931).

Petre (1906) se refirió en su crónica de la calidad del café colombiano y a su importancia en el sector agrícola mundial, al señalar que la región del Tolima estaba conectada al recién instalado sistema ferroviario entre Ambalema y La Dorada, para la exportación del grano. Asimismo, al noreste del Tolima se establecieron grandes plantaciones de café como las haciendas de Arabia, La Aurora, Nuevo Mundo, Varsovia y Valparaíso (Ramírez, 2002; FNCC, 2019).

Para ese entonces, la hacienda cafetera representaba un atributo fundamental del paisaje y de la unidad familiar campesina, que por definición se orientaba a la reproducción de la subsistencia y a las decisiones relacionadas con el manejo de la naturaleza. La hacienda constituye el motor del desarrollo cafetero e indirectamente del capitalismo en Colombia (Palacios, 1980). Con el tiempo, el conjunto de fincas configurará la forma de las veredas. 
Figura 3. El Tolima y la Ecorregión Cafetera

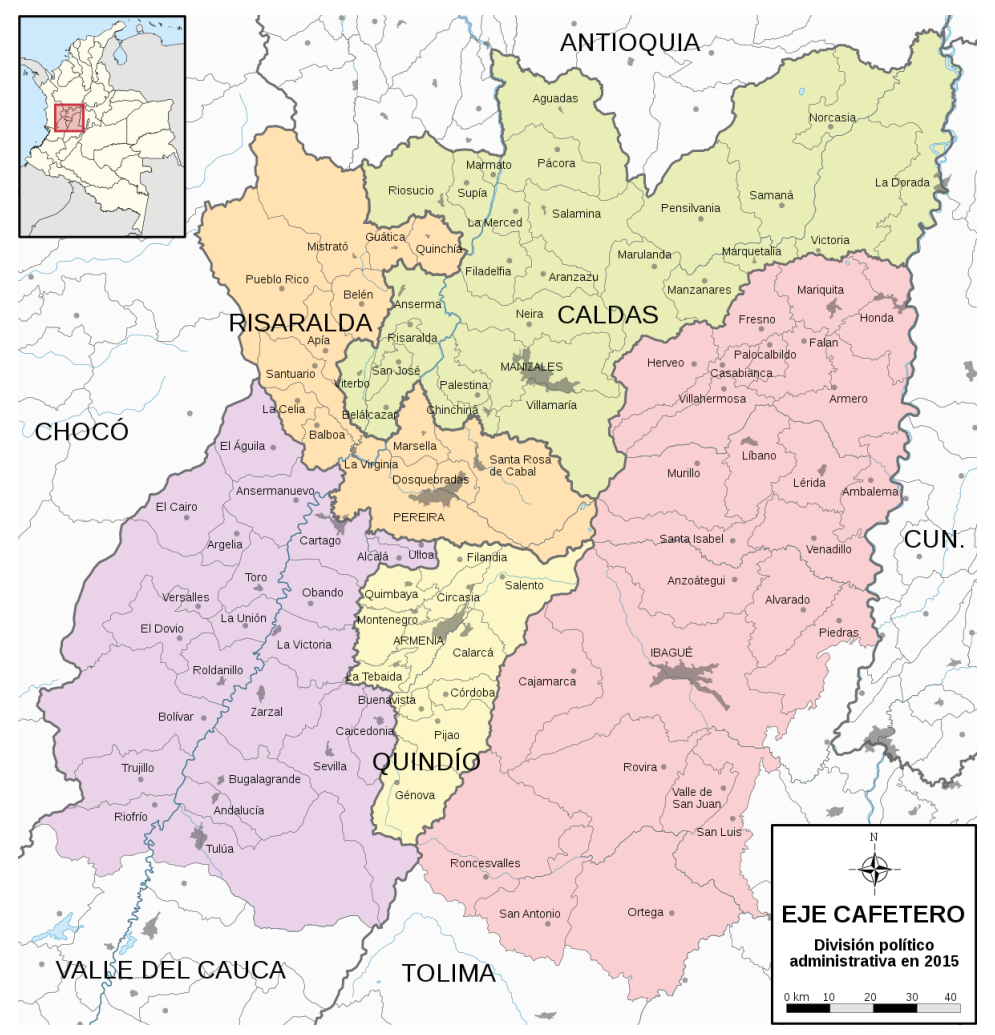

Fuente: Licencia internacional de Creative Commons Reconocimiento-Attribution-Share Alike 4.0

Además, el Parque Nacional Natural de Los Nevados: "Comprende una superficie aproximada de 58300 hectáreas y se encuentra en los municipios de Anzoátegui, Casabianca, Herveo, Ibagué, Murillo, Santa Isabel y Villahermosa" (Cortolima, 2014, p. 107). Sus ecosistemas vinculados al paisaje cultural cafetero del Tolima cuentan con un gran número de bosques nativos "considerados estratégicos para la conservación de la biodiversidad global" (Velandia, 2018, p. 52). Los Andes sudamericanos son uno de los hotspots de biodiversidad más importantes del mundo, denominado "Hotspot de los Andes Tropicales”. Según el Fondo de Cooperación para los Ecosistemas Críticos (CEPF), este hotspot es uno de los de mayor riqueza y endemismo de especies (Velandia, 2018). De la flora endémica como los bosques de palmeras de cera (Ceroxylon quindiuense), el Frailejón (espeletia), extensiones en páramos, fauna de una gran variedad de anfibios, aves, 
especialmente el colibrí cabecicastaño (Anthocephala berlepschi) y el loro orejiamarillo (Ognorhynchus icterotis) entre las especies de mamíferos y la diversidad de reptiles. Además, los bosques gigantes de bambú o guadua (Angustifolia Kunth) también están protegidos y condicionados para su uso sostenible (Cortolima, 2014).

El paisaje cultural cafetero del Tolima se ubica en la zona de vida subandina (1 100-2350 m.s.n.m.) (Holdridge, 1992), configurada por una estructura ecológica primaria por su clima, suelos volcánicos, fuentes de agua y diversidad biológica. Las principales amenazas naturales han sido identificadas por los movimientos en masa, las inundaciones, las erupciones volcánicas y sísmicas, y los riesgos de incendios forestales y deslizamientos de tierra. El territorio del paisaje cultural cafetero del Tolima tiene un alto riesgo sísmico, debido a la triple unión que se produce en la esquina de la placa tectónica sudamericana donde convergen las placas de Nazca y del Caribe. La región está afectada por cinco volcanes activos pertenecientes al Complejo Volcánico Machín-Cerro Bravo, en el lado oriental de la cordillera Central (Velandia, 2018; SGC, 2012) (Véase la Figura 4).

Figura 4. Fotocomposición del paisaje cultural cafetero de Planadas, Tolima

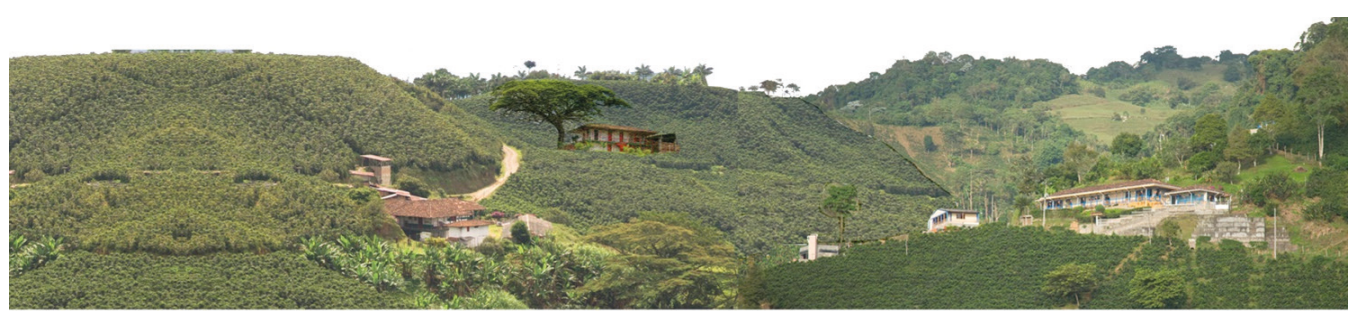

Fuente: Velandia (2018, p. 57)

En la región del paisaje cultural cafetero del Tolima, Gómez et al. (1991), delimitaron microrregiones agroecológicas o "ecotopos cafeteros", clasificados como 206B, 207B, 208B, 209B, 210B, 211B, 315A, 316A, 317A, especificadas en la topografía, la altitud, el tipo de cultivo de café, las precipitaciones, el tipo de suelos y los minerales. Esta clasificación corresponde a la $2 \mathrm{~B}$ cordillera Central - vertiente oriental y $3 \mathrm{~A}$ cordillera Oriental - vertiente occidental.

El café del Tolima se encuentra en un rango de altitud entre "900 y 2100 metros sobre

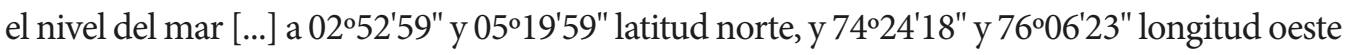
en el piedemonte oriental y occidental del valle del río Magdalena" (Velandia, 2018, p. 50). 
Pertenece a la zona de vida subandina de Holdridge o bosque premontano muy húmedo (bmh-PM) situado en zonas de ladera, con precipitaciones que oscilan entre 2 000-4 $000 \mathrm{~mm}$ y temperaturas entre $18-24{ }^{\circ} \mathrm{C}$, características de un clima muy húmedo. También presenta las zonas de vida del bosque muy húmedo de baja montaña (bmh-MB), situado en las formaciones montañosas enmarcadas por los páramos y el suelo premontano. Se encuentra en zonas donde la temperatura fluctúa entre 12 y $18^{\circ} \mathrm{C}$, y las precipitaciones varían entre 2000 y $4000 \mathrm{~mm}$, condiciones típicas del clima frío muy húmedo. (Cortolima, 2014, p. 34).

[A lo largo del año] la época de floración en el paisaje cafetero define dos zonas con épocas de cosecha diferentes. La primera (en el $75 \%$ de los municipios) se cosecha entre los períodos de marzo y junio-octubre y noviembre (Velandia, 2018, p. 50).

\section{Resultados}

Para la construcción de una agenda del paisaje cultural cafetero del Tolima, debido a que está compuesto por atributos cuantitativos y cualitativos, se logró un modelo en un Sistema de Información Geográfico (SIG) a partir del mapeo de los atributos cuantitativos para la delimitación. Utilizando el motor ArcGis 10.3 de ESRI, se desarrolló a partir de una geodatabase (FNCC, 2019) e imágenes Geotiff (Georeferenced Tagged Image File Format obtenidas de https://earthexplorer.usgs.gov/). Mediante el procesamiento de las herramientas Sistema de Información Geográfico (SIG), los rasgos fisiográficos se modelaron por elevación digital, curvas de nivel y luego se procesó mediante análisis algorítmico de sombreado y pendientes, recuperados a partir de valores de datos y clases ordenados por dos grandes rangos: datos de altitud más pendientes altas (45\%) entre 1200-1 800 m.s.n.m., y pendientes medias (30 \%) entre 1000-1200 m s. n. m. y 1800 2000 m s. n. m. Finalmente, el modelo de delimitación y de los rangos mencionados, se constituyó por 2 zonas: la zona principal que concentra el mayor número de atributos con una superficie de 339698 hectáreas; y la zona de amortiguamiento con una superficie de 231626 hectáreas (Ver figuras 5 y 6). 
Figura 5. Modelo digital de elevación, análisis de pendientes y sombreado de colinas

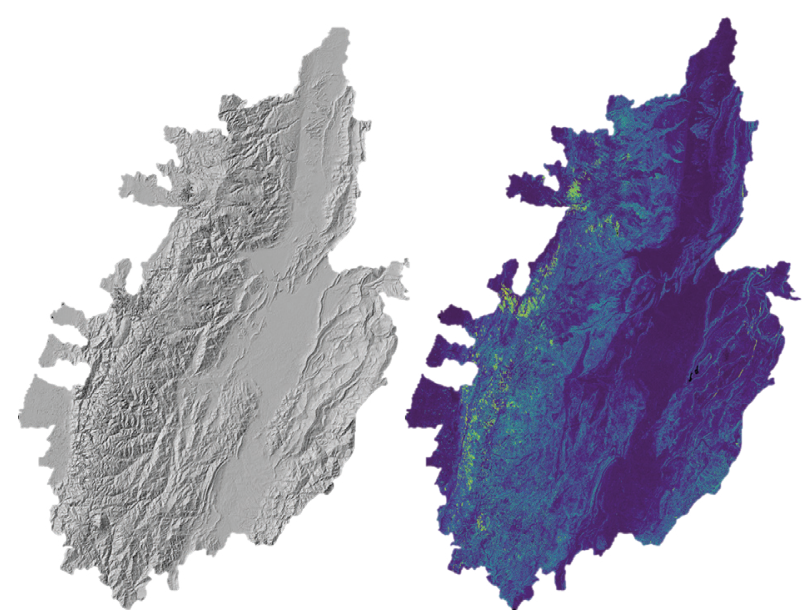

Fuente: elaborada por César Augusto Velandia Silva, 2019

Figura 6. Delimitación del paisaje cultural cafetero del Tolima: Zona principal-zona de amortiguamiento y veredas (unidades territoriales)

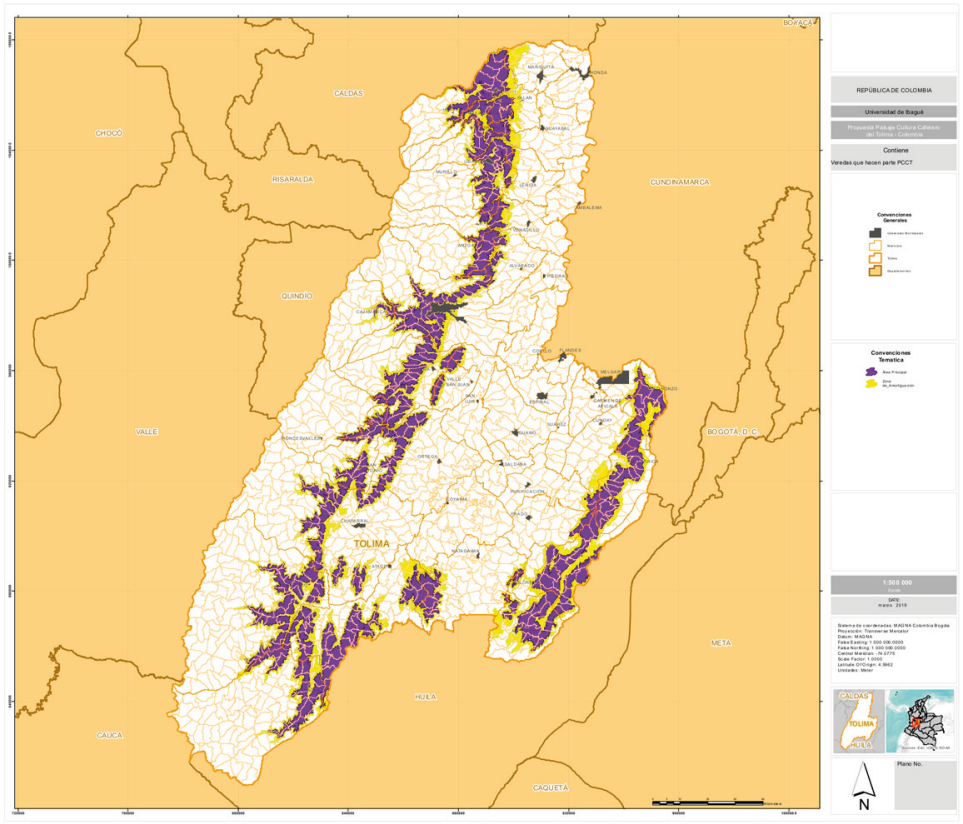

Fuente: elaborada por César Augusto Velandia Silva, 2019 
Logrado lo anterior, debe formularse la agenda social básica (general). A continuación, se proponen los siguientes 8 pasos:

- Validar la delimitación con la comunidad y el Gobierno, y elevarla a una política regional del Tolima (de acuerdo con el marco legal).

- Establecer un comité para convocar a las partes interesadas (stakeholders).

- Desarrollar procesos con la comunidad para complementar la valoración del patrimonio contenido en la delimitación.

- Aplicar herramientas participativas (mapeo social) con la comunidad para identificar las aspiraciones y desafíos del paisaje valorado.

- Compartir y acordar los desafíos y objetivos de un programa de gestión.

- Aprobar por el comité y luego ejecutar el programa de gestión y el seguimiento.

- Medir los indicadores de progreso establecidos en la agenda, difundirlos y discutir sus logros.

- Evaluar la agenda cada 3 o 5 años para la mejora continua.

\section{Potencial uso}

Se propuso una agenda de gestión (específica) de 6 temas claves, para definir estrategias que promuevan herramientas de participación con las comunidades, para que la organización social asuma su participación y responsabilidad en la valorización del paisaje. Será importante compartir la metodología con la comunidad, para que entiendan que se trata de un proceso de conocimiento que proviene de un estudio en profundidad de los vínculos singulares naturaleza-cultura con ellos (los datos arqueológicos, los sistemas de cultura simbólico-volcánica o microverticalidad), y de establecer las interrelaciones sociales entre los ecosistemas (naturaleza) y el patrimonio (cultura) al que contribuirán con sus valiosos aportes. Previendo esto, se propuso lo siguiente:

- Construir un conocimiento compartido con las comunidades: generando una metodología propia de valorización sobre el paisaje cultural cafetero del Tolima, construida a partir de la condición antigua como lo demuestra el Instituto Nacional de Patrimonio Cultural-INPC (2015) y el estudio del origen del espacio prehispánico con la ayuda de un equipo de expertos y el Gobierno departamental (INPC, 2014).

- Declarar la delimitación del paisaje cultural cafetero del Tolima como bien de interés cultural del ámbito departamental y promover una política regional para su conservación y difusión a través de la educación pública por parte del Gobierno del Tolima. 
- Desarrollar un Sistema de Información Geográfico (SIG) para la gestión de la planificación: permitir la definición de reglas de determinación del territorio que sirvan de base a instrumentos normativos para la gestión de la agrobiodiversidad. Según los planes de gestión de la Ley colombiana, solo es posible para el uso de la tierra, los planes rurales de seguridad alimentaria, la protección del patrimonio y las reservas ambientales. Reforzar la gestión de la base agroproductiva garantizará la conservación de los valores materiales e inmateriales.

- Reforzar el proceso de posconflicto: incluir acuerdos de paz sobre reforma agraria, equidad y justicia en el retorno de la tierra a las personas desplazadas por el conflicto (Molano, 2015) y se permite una nueva política de desarrollo agrícola en una Reforma Rural Integral (RRI) para el desarrollo de la agricultura campesina, familiar y comunitaria (Isaza y Velandia, 2018).

- Mejorar las prácticas de cultivo sostenibles para el cambio climático: recientemente se puede revisar una contribución como respuesta política a su vulnerabilidad en los sitios de patrimonio mundial (Markham, 2018), para que tenga implicaciones regionales como en el Tolima. En el plano nacional, Colombia ha desarrollado acciones de política a partir de la iniciativa "Café Climático Inteligente" (Uribe y Velandia, 2013, p. 143) y la "Estrategia de Sostenibilidad 2027" (Perfect Daily Grind, 2015; Isaza y Velandia, 2018, p. 237).

- Reforzar las posibles iniciativas locales para el desarrollo de la formulación de políticas en materia de cultura y educación: los gobiernos locales y las organizaciones sociales tienen una falta de cohesión a través de la cultura y la paz. Construir el conocimiento del patrimonio del paisaje cultural cafetero del Tolima será un fuerte valor educativo y de apropiación para la identidad y la reconciliación entre indígenas, grupos afrocolombianos desplazados, campesinos y exguerrilleros entre otros. En este sentido, las soluciones deben intensificar la transmisión de los valores del paisaje cultural cafetero del Tolima a las próximas generaciones.

\subsection{Aspectos claves para el manejo}

Al tener como referencia el plan de manejo (Mincultura-FNCC, 2009), el primer tema de interés es el desarrollo sostenible. La visión general resultante de una reflexión sobre los valores en el paisaje cultural cafetero de Colombia estará obligada a responder a la seguridad alimentaria, a las prácticas de agricultura sostenible y a las medidas de conservación del suelo y del agua, entre otras. 
INDAGA]ZE e-ISSN: 2357-5042 • Número 8 (2020) • Universidad de Ibagué • doi: https://doi.org/10.35707/indagare/808

Estos se convertirán en los temas claves de una agenda de manejo. Pueden aplicarse sobre la base de los conocimientos adquiridos a través del mencionado proceso social de ocho etapas. El Tolima, como región con calidad agrícola y de producción de café a nivel nacional, necesita una agenda de manejo del paisaje cultural cafetero del Tolima como una oportunidad para articular la dimensión cultural del territorio y permitir una mejora en la calidad de la vida rural de su población.

El fortalecimiento de los vínculos culturales de las comunidades con el territorio es esencial para el conocimiento tradicional de las montañas, la mejora de las condiciones sociales y la cohesión con la política de paz de las comunidades del Tolima. Por lo tanto, la protección del paisaje cultural cafetero del Tolima se convierte en un elemento clave para el desarrollo socioambiental vinculado y en un eje articulador de las iniciativas regionales de conservación de la naturaleza.

Colombia ha potenciado una política nacional desde la conservación del paisaje cultural a través del CONPES (DNP, 2014). Desde allí, el Tolima puede apoyar lo anterior en una política regional y operar acciones con participación de las secretarías de Ambiente, Educación, Cultura y Desarrollo Rural. Por lo tanto, es necesario contar con la voluntad y el interés de los políticos para innovar en los procesos propuestos y aprovechar el potencial cultural-natural de nuestra región cafetera.

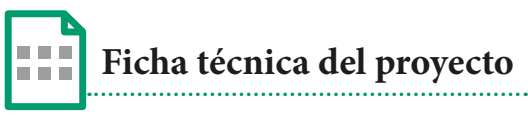

Título del proyecto: Estudio de valoración del paisaje cultural cafetero del Tolima como Bien de Interés Cultural.

PRIT: Desarrollo regional inclusivo y sustentable.

Código del proyecto: $18-560-$ INT.

Palabras claves: Paisaje cultural, cultura cafetera, bien de interés cultural.

Grupo de investigación: Rastro Urbano.

Investigador principal: César Augusto Velandia Silva.

Correo electrónico: cesar.velandia@unibague.edu.co

\section{Referencias}

Anschuetz, K., Wilshusen, R. H., \& Sheick, C. L. (2001). An archaeology of landscapes: Perspectives and directions. Journal Archaeological Research, 9(2), 157-211. https://doi.org/10.1023/A:1016621326415 Argüello, P. (2016). Arqueología regional en el valle de Tena: Un estudio sobre la microverticalidad Muisca. Antípoda, (25), 143-166. https://doi.org/10.7440/antipoda25.2016.07 
Bergquist C. W. (1978). Coffee and conflict in Colombia, 1886-1910. Durham, Estados Unidos: Duke University Press.

Cano, M. \& López, C. (2017). Impacto de las erupciones volcánicas durante los últimos 12.000 años en la región del Cauca Medio, centro-occidente de Colombia: Aportes de la geoarqueología y gestión del riesgo al conocimiento de interacciones milenarias naturaleza-cultura. En: Ugalde, M. (Ed.), Volcanes, Cenizas y Ocupaciones Antiguas en Perspectiva Geoarqueológica en América Latina, (pp. 42-54). Quito, Ecuador: PUCE.

Corporación Autónoma Regional de Risaralda. Carder (2002). Ecorregión Eje Cafetero: Un territorio de oportunidades. Pereira, Colombia: Carder. Recuperado de https://bit.ly/2Kf08wE

Cleere, H. (1995). Cultural landscapes as world heritage. Conservation and Management Archaeology Studies, 1 (1), 63-68. https://doi.org/10.1179/135050395793137171

Corporación Autónoma Regional del Tolima. Cortolima (2014). Atlas ambiental del Tolima. Ibagué, Colombia: El Nuevo Día. Recuperado de https://www.cortolima.gov.co/sites/default/files/atlas/\#p=1 Cosgrove, D. (1984). Social formation and symbolic landscape. Londres, Inglaterra: Croom Helm.

Cosgrove, D. (1985). Prospect, perspective and the evolution of the landscape idea. Transactions of the Institute of British Geographers, 10 (1), 45-62. https://doi.org/10.2307/622249

Cosgrove, D. (1994). Cultural landscape. En Johnston, R., Gregory, D., Pratt, G., Watts, M. \& Whatmore, S. (Eds.), The Dictionary of Human Geography (pp. 114-115). Oxford, Inglaterra: Blackwell.

Cosgrove, D. \& Daniels, S. (1988). The iconography of landscape: Essays on the symbolic representation. Design and use past environments. Cambridge, Inglaterra: Cambridge University Press.

Cuéllar, A. (2009). The Quijos chiefdoms: Social change and agriculture in the Eastern Andes in Ecuador. Pittsburg-Bogotá: University of Pittsburg-Universidad de Los Andes-Ministerio de Cultura del Ecuador.

Cuéllar, A. (2011). Modelos económicos para las vertientes orientales de los Andes. El caso de los cacicazgos quijos desde una perspectiva arqueológica. Indiana, 28, 35-57. http://dx.doi.org/10.18441/ind. v28i0.35-57

CEPF. Fondo de Colaboración para los Ecosistemas Críticos (2015). Ecosystem profile. Tropical Andes Biodiversity Hotspot. CEPF.

Deetz, J. (1990). Landscapes as cultural statements. En Kelso W., Most R., (Eds.), Earth Patterns: Essays in Landscape Archaeology (pp. 1-4). Charlottesville, Estados Unidos: University Press of Virginia.

Dickau, R., Aceituno, F., Loaiza, N., López, C., Cano, M., Herrera, L., Restrepo, C. \& Ranere, A. (2015). Radiocarbon chronology of terminal Pleistocene to middle Holocene human occupation in the Middle Cauca Valley, Colombia. Quaternary International, 363, 43-54. https://doi.org/10.1016/j. quaint.2014.12.025

Departamento Nacional de Planeación. DNP (2014). Conpes $n^{\circ}$ 03808. Politica para la preservación del Paisaje Cultural Cafetero. Bogotá, Colombia: DNP. Recuperado de https://bit.ly/3nrE4NP

Federación Nacional de Cafeteros de Colombia. FNCC (2019). Atlas de la caficultura en Colombia. Bogotá, Colombia: FNCC.

Federación Nacional de Cafeteros de Colombia. FNCC (2015). Denominación de origen Café del Tolima. Documento de trabajo. Bogotá, Colombia: FNCC.

Federación Nacional de Cafeteros de Colombia. FNCC. Huila (s. f.). Recuperado de https://huila. federaciondecafeteros.org 
INDAGA3E e-ISSN: 2357-5042 • Número 8 (2020) • Universidad de Ibagué • doi: https://doi.org/10.35707/indagare/808

Georeferenced Tagged Image File Format (s.f.) USGS. Recuperado de https://earthexplorer.usgs.gov/ Gómez, G., Caballero, R \& Baldion, R. (1991). Ecotopos cafeteros de Colombia. Bogotá, Colombia: FNCC. Guhl, A. (2008). Café y cambio de paisaje en Colombia, 1970-2005. Medellín, Colombia: EAFIT- Banco de la República. Recuperado de https://www.halacsolcha.org/index.php/halac/article/view/162/157

Hall, C., Macgowan, H. \& Bynum, M. (1931). The coffee industry in Colombia. Washington: Government Printing Office.

Hirsch, E. \& O'Hanlon, M. (Eds.). (1995). The Anthropology of landscape: Perspectives on place and space. Oxford, Inglaterra: Clarendon Press.

Holdridge, L. (1992). Life zone ecology. San José, Costa Rica: Centro de Ciencia Tropical.

Instituto Nacional de Patrimonio Cultural. INPC (2014). Estudios multidisciplinarios en cinco espacios prehispánicos tardíos del Ecuador. Quito, Ecuador: INPC-Universidad de Cuenca.

Instituto Nacional de Patrimonio Cultural. INPC (2015). Guía metodológica para el paisaje cultural ecuatoriano. Quito, Ecuador: INPC-Universidad de Querétaro.

Ingold, T. (1993). The temporality of landscape. World Archaeology. 25 (2), 152-174. https://doi.org/10.108 $0 / 00438243.1993 .9980235$

Isaza, J. (2014). Presentación. En Isaza, J. (Ed.). Patrimonio cultural y academia en Colombia (pp. 1-10). Bogotá, Colombia: Universidad de los Andes.

Isaza, J. \& Velandia, C. (2018). Sustainable development and nature-culture linkages in the Coffee Cultural Landscape of Colombia. En Larsen, P. \& Logan, W. (Eds.). World Heritage and Sustainable Development: New Directions in World Heritage Management (pp. 226-239). Londres, Inglaterra y Nueva York, Estados Unidos: Routledge.

Larsen, P. \& Wijesuriya, G. (abril, 2015). Nature and culture interlinkages in the World Heritage. World Heritage-Nature-Culture Links, pp. 4-15. Recuperado de http://whc.Unesco.org/en/review/75

Markham, A. (2018). A comprehensive policy response to climate change vulnerability in World Heritage sites. En World Heritage Watch, World Heritage Watch Report 2018 (pp. 10-12). Berlín, Alemania: World Heritage Watch.

Mincultura (2019). Decreto número 2358 de 2019, por el cual se modifica y adiciona el Decreto 1080 de 2015 reglamentario del Sector Cultura, en lo relacionado con el Patrimonio Cultural Material e Inmaterial. Diario Oficial No 51.178. 26 de diciembre. Recuperado de https://bit.ly/2KDJ3MI

Mincultura \& Federación Nacional de Cafeteros de Colombia. FNCC. (2009). Plan de manejo y protección del Paisaje Cultural Cafetero. Bogotá, Colombia: Ministerio de Cultura, FNCC.

Mincultura \& Federación Nacional de Cafeteros de Colombia FNCC. (2016). Paisaje Cultural Cafetero de Colombia: Fusión excepcional entre naturaleza, cultura y trabajo colectivo. Bogotá, Colombia: Ministerio de Cultura-FNCC. Recuperado de https://bit.ly/2WoSbYi

Ministerio de Cultura del Perú (2017). Paisajes culturales en América Latina. Lima, Perú: Ministerio de Cultura. Recuperado de http://repositorio.cultura.gob.pe/handle/CULTURA/637

Molano, A. (2015). Fragmentos de la historia del conflicto armado (1920-2010). Bogotá, Colombia: Espacio Crítico.

Mujica, E. \& Holle, M. (2002). Los Andes y la transformación cultural del paisaje. Quito, Ecuador: Unesco.

Murra, J. (1974, octubre). Límites y limitaciones del "Archipiélago Vertical" en los Andes. Segundo Congreso Peruano del Hombre y la Cultura Andina. Trujillo, Perú. Recuperado de https://revistas. unal.edu.co/index.php/maguare/article/download/93-98/16437 
Murra, J. (1975). Formaciones económicas y políticas del mundo andino. Lima, Perú: Instituto de Estudios Peruanos-IEP Ediciones.

Norton, W. (1989). Explorations in the understanding of landscape: A cultural geography. Nueva York, Estados Unidos: Praeger.

Palacios, M. (1980). Coffee in Colombia, 1850-1970: An economic, social and political history. Cambridge, Inglaterra: Cambridge University Press.

Perfect Daily Grind (25 de abril de 2015). Colombia tiene una nueva meta: Café sostenible para el 2027. Recuperado de https://bit.ly/38hBK5v

Petre, F. L. (1906). The Republic of Colombia: An account of the country, its people, its institutions and its resources. Londres, Inglaterra: E. Stanford. Recuperado de https://hdl.handle.net/2027/ txu.059173023659751

Plachter, H. \& Rössler, M. (1995). Cultural landscapes: Reconnecting culture and nature. En Von Droste B, Plachter, H. \& Rössler, M. (Eds.). Cultural Landscapes of Universal Value (pp. 15-18). Munich, Alemania: Jena Fischer Verlag.

Quattrin, D. (2001). Prehispanic chiefdoms in the Valle de la Plata, Vol 4. Vertical economy, interchange and social change during the formative period. Pittsburg, Estados Unidos-Bogotá, Colombia: University of Pittsburg-Universidad de los Andes. Recuperado de http://www.pitt.edu/ ccapubs/pdfdownloads/ PITTmem11-Quattrin_2001.pdf

Ramírez R. (2008). Historia laboral de una hacienda cafetería. Hacienda La Aurora, Tolima-Colombia, 1882-1982.Medellín, Colombia: Universidad Nacional de Colombia-La Carreta Editores.

República de Colombia. Ley 1185 de 2008. Por la cual se modifica la Ley 397 de 1997 y se agrega la Ley General de Cultura. Diario Oficial No 46.929 (12 de marzo). Recuperado de http://www. secretariasenado.gov.co/senado/basedoc/ley_1185_2008.html

Rincón, F. (Ed.). (2016). La caficultura como patrimonio cultural, social y productivo. Manizales, Colombia: Ministerio de Cultura-FNCC.

Rössler, M. (febrero, 1993). The integration of cultural landscapes into the world heritage, conserving outstanding cultural landscapes, protecting outstanding cultural landscapes. World Heritage Newsletter. Recuperado de http://whc.unesco.org/en/newsletter/1/

Rössler, M. (2003). Linking nature and culture: world heritage cultural landscapes. En UNESCO World Heritage Center, World Heritage Papers Cultural Landscapes: The Challenges of Conservation (pp. 10-15). Ferrara, Italia: Unesco. Recuperado de http://whc.unesco.org/documents/publi_wh_ papers_07_en.pdf

Rössler, M. \& Tricaud, P. M. (Eds.). (2009). World heritage cultural landscapes: A handbook for conservation and management. Recuperado de http://whc.unesco.org/documents/publi_wh_papers_26_en.pdf

Salgado, H. \& Varón, M. (2019). Early prehispanic settlement in the Magdalena Valley in Tolima, Colombia. Balance and perspectives. Quaternary International, 505 (15), 55-68. https://doi.org/10.1016/j. quaint.2018.04.046

Servicio Geológico Colombiano. SGC. (2012). Informe de actividad del Complejo Volcánico Cerro BravoCerro Machín. Manizales, Colombia: SGC.

Unesco (2008). Directrices operacionales para la aplicación de la Convención del Patrimonio Mundial. París, Francia: Centro del Patrimonio Mundial. Recuperado de http://whc.unesco.org/archive/ opguide08-es.pdf 
INDAGA3E e-ISSN: 2357-5042 • Número 8 (2020) • Universidad de Ibagué • doi: https://doi.org/10.35707/indagare/808

Unesco (s.f.) El paisaje cultural del café de Colombia. Recuperado de https://whc.unesco.org/en/list/1121 Unesco. (2017). Directrices operacionales para la aplicación de la Convención del Patrimonio Mundial. París, Francia: Centro del Patrimonio Mundial. Recuperado de http://whc.unesco.org/archive/opguide08es.pdf.

Uribe, S., \& Velandia, C. (2013). Naturaleza, cultura y productividad. Gestión del Paisaje Cultural Cafetero de Colombia. En Niño N. \& Saldaña, J. (Eds.), III Congreso Internacional en Patrimonio y Desarrollo Sustentable (Pydes 2013) (pp. 141-143). Ciudad de México, México: Universidad Autónoma de Guerrero-Editorial Praxis.

Velandia, C. (2017). Indigenous landscapes Coffee Cultural Landscape of Colombia. En Inaba N., Ishizawa, M. \& Yoshida, M. (Eds.), Journal of World Heritage Studies Special Issue: Proceedings of the First Capacity Building Workshop on Nature-Culture Linkages in Heritage Conservation in Asia and the Pacific (CBWNCL 2016) (pp. 44-50). Tokio, Japón: Universidad de Tsukuba.

Velandia, C. (2018). La valoración patrimonial del paisaje cultural cafetero del Tolima, Colombia. Retos y estrategias para su conservación y apropiación. Identidades: Territorio Cultura Patrimonio, 7, 43-62. Recuperado de https://upcommons.upc.edu/handle/2117/130555.

Von Droste, B., Plachter, H. \& Rössler, M. (1995). Cultural landscapes of universal value: Components of a global strategy. Stuttgart, Alemania: Gustav Fischer Verlag.

Wagstaff, J. (1987). Landscape and culture: Geographical and archaeological perspectives. Oxford, Inglaterra: Blackwell. 